\begin{abstract}
Ángel Herraiz Adillo $0^{1, *}$
José Javier Romero Parrilla²

1. Centro de Salud de Tragacete. Servicio de Salud de Castilla-La Mancha (SESCAM). Cuenca. España.

2. Centro de Salud de Tarancón. Servicio de Salud de Castilla-La Mancha (SESCAM). Cuenca. España.
\end{abstract}

${ }^{*}$ Autor para correspondencia.

Correo electrónico: ahadillo@hotmail.com (Ángel Herraiz Adillo).

Recibido el 1 de octubre de 2020; aceptado el 14 de octubre de 2020

\section{Prevalencia de úlceras por presión en atención primaria: estudio de Cuenca}

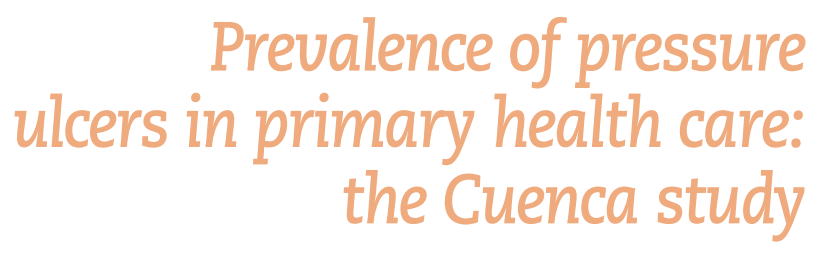

\section{RESUMEN}

Objetivos: 1) Establecer la prevalencia puntual de úlceras por presión (UPP) y 2) caracterizar los pacientes afectados y las lesiones identificadas. Metodología: Estudio observacional, descriptivo y transversal, realizado en la Gerencia de Atención Integrada de Cuenca (España). Se remitió cuestionario online a todos los profesionales de atención primaria, recogiendo variables epidemiológicas y clínicas de los pacientes afectados por UPP, así como datos de la propia lesión y el tratamiento. Resultados: Contestaron 152 profesionales (tasa de respuesta $=98,1 \%$ ), lo que permitió estudiar a 131190 habitantes. En total, se contabilizaron 72 lesiones por UPP que afectaban a 51 pacientes (edad $=86,6 \pm 10,4$ años), lo que representa una prevalencia global de 0,389\%o (IC95\%: 0,296-0,511). La prevalencia total fue mayor en mujeres que en hombres $(0,532 \%$ vs. $0,245 \%, p=0,008$, respectivamente), y en ambos grupos la prevalencia aumentó al incrementarse la edad, con prevalencias en individuos de 65 años o mayores del 1,982\% y 1,144\%o para mujeres y hombres, respectivamente. Hasta en el 32,1\% y 43,2\% de los casos no se usó escala de valoración del riesgo y superficie especial de manejo de la presión, respectivamente. Un 47,2\% de los pacientes presentó lesiones múltiples, y un $97,2 \%$ de las UPP presentó una categoría > 1, utilizándose la cura en ambiente húmedo en el 59,7\% de las lesiones. Conclusiones: la prevalencia de UPP en una provincia con alto componente rural fue baja, si bien la distribución por edad y sexo y el perfil de las lesiones guardan analogía con los de otros estudios nacionales. El uso de estrategias preventivas y tratamientos con evidencia contrastada continúa siendo bajo.

PALABRAS CLAVE: Atención primaria, enfermería, prevalencia, úlcera por presión.

\section{ABSTRACT}

Objectives: 1) To determine the point prevalence of pressure ulcers (PU) and 2) to characterize the identified patients and lesions. Methods: Observational, descriptive and cross-sectional study, settled in the "Gerencia de Atención Integrada" of Cuenca (Spain). An ad hoc online questionnaire was sent to all nurses attending Primary Care centres, and epidemiologic and clinic variables related to patients with PU were recorded. Results: In total, 152 professionals (response rate 98.1\%) answered the questionnaire, recording data of 131,190 habitants. We identified 72 PUs from 51 patients ( $86.6 \pm 10.4$ years), which represents an overall prevalence of $0.389 \%$ (IC 95\%: 0.296-0.511). The global prevalence was higher in women than men $(0.532 \%$ vs. $0.245 \%$ o, $\mathrm{p}=0,008$, respectively) and increased with age, finding a prevalence in patients $\geq 65$ years old of $1.982 \% 0$ and $1.144 \%$ o for women and men, respectively. A risk assessment scale for PU prevention was not used in up to $32.1 \%$ of patients, and $43.2 \%$ of patients did not use any pressure relieving support surface. Multiple lesions were identified in $47.2 \%$ of patients, and $97.2 \%$ of PUs had a category $>1$. A wet wound healing therapy was used in $59.7 \%$ of the lesions. Conclusions: Overall, the prevalence of PU found in a rural province was low, but the distribution of age and gender as well as the profile of the lesions were like those found in national studies. The use of preventive strategies and evidence-based treatments is still low.

KEYWORDS: Primary care; nursing; prevalence; pressure ulcer.

\section{У INTRODUCCIÓN}

Las úlceras por presión (UPP) son lesiones localizadas en la piel o tejidos subyacentes, habitualmente sobre una prominencia ósea, como resultado de la presión, pudiéndose asociar además fuerzas de cizalla ${ }^{1}$. El Grupo Nacional para el Estudio y Asesoramiento en Úlceras por Presión y Heridas Crónicas (GNEAUPP) ha reclamado desde su creación en 1994 que "las UPP, lejos de ser un proceso banal, inevitable o silente, son un problema de salud de primer orden a nivel mundial”. Así, en España, el $4^{\circ}$ Estudio Nacional de Prevalencia del GNEAUPP encontró que en la asistencia hospitalaria, la prevalencia de UPP llegaba al 7,9\% y que esta subía hasta el $18,0 \%$ y $13,4 \%$ en las unidades de cuidados intensivos y residencias sociosanitarias, respectivamente, constituyendo, aún en el siglo XXI, una "auténtica epidemia bajo las sábanas"2.

Además de esta alta prevalencia, las UPP implican una importante morbimortalidad. Un amplio estudio norteamericano mostró que presentar una UPP aumentaba el riesgo de muerte en 5 veces en los pacientes ingresados ${ }^{3}$, y a nivel nacional se han notificado tasas ajustadas de mortalidad de hasta 20 y 31 defunciones por cada 100000 habitantes en hombres y mujeres, respectivamente ${ }^{4}$. Igualmente, las UPP son fuente de gran sufrimiento para 
los pacientes afectados y sus familiares tanto en el plano físico y emocional $^{5}$ como social ${ }^{6}$, a causa del dolor, mal olor, desfiguración, pérdida de la intimidad y autoestima o cambios en el rol social que el propio paciente o cuidador deben asumir. Asimismo, las UPP son costosas, generando el 4\% y $5 \%$ del gasto sanitario en Europa ${ }^{7}$ y España ${ }^{8}$, respectivamente.

Aunque la prevalencia de UPP a nivel hospitalario ha sido bien estudiada, pocos estudios a nivel internacional han analizado dicha prevalencia en el contexto de la atención primaria, existiendo además importante heterogeneidad en los resultados ${ }^{2,9}$. A nivel nacional, el GNEAUPP viene realizando desde el año 2001 estudios epidemiológicos cada 4 años que analizan el problema en los diferentes niveles asistenciales ${ }^{2,10-12}$. Sin embargo, características propias de nuestra provincia como son el gran desarrollo rural, gran dispersión geográfica y baja densidad poblacional, así como la inexistencia de un servicio de referencia en heridas complejas, que es una estructura que sí está desarrollada en poblaciones de mayor tamaño, limitarían la extrapolación de esos resultados a nuestra provincia.

Conocer la situación actual en los centros de salud de Cuenca nos situaría dentro del mapa nacional y permitiría la evaluación de aquellas medidas preventivas o terapéuticas que pudieran ponerse en marcha a partir del presente estudio.

\section{$\searrow$ OBJETIVOS}

Los objetivos que se plantean son los siguientes:

1. Determinar la prevalencia puntual de UPP en la provincia de Cuenca en el contexto de atención primaria.

2. Identificar las características de los pacientes afectados por UPP.

3. Caracterizar dichas lesiones y el tratamiento empleado en las mismas.

\section{У MÉTODOS}

\section{Tipo de estudio}

Estudio observacional, transversal, descriptivo, de prevalencia puntual mediante cuestionario online.

\section{Población de estudio}

La población diana está compuesta por todos los sujetos adscritos a los centros de salud pertenecientes a la Gerencia de Atención Integrada (GAI) de Cuenca (32 centros, 27 rurales y 5 urbanos o con población mayor de 10000 habitantes), todos ellos de titularidad pública.

Este estudio forma parte de un proyecto más amplio denominado "Estudio de Prevalencia de Heridas Crónicas de la Gerencia de Atención Integrada de Cuenca 2020".

\section{Criterios de inclusión}

\section{Presentar UPP.}

2. Estar adscrito y recibir cuidados (tanto en consulta como en domicilio) por una UPP en cualquier centro de salud de la GAI de Cuenca.

No se determinaron criterios de exclusión.

\section{Recogida de datos}

Se elaboró un cuestionario ad hoc a través de la plataforma Google Forms. Aunque el cuestionario fue pilotado en una muestra de 10 enferme- ros, incluyendo las sugerencias aportadas y corrigiendo errores, dicho cuestionario no está validado. Posteriormente, dicho cuestionario fue enviado a través de correo corporativo a la totalidad de los profesionales de enfermería de atención primaria de la GAI de Cuenca $(\mathrm{n}=155)$; ver Material suplementario 1, cuestionario Google Forms.

Al tratarse de un estudio de prevalencia puntual, los profesionales debían contestar sobre aquellos pacientes con UPP que tenían a fecha de 17 de febrero de 2020, aunque la encuesta estuvo abierta durante 25 días. Las duplicidades entre pacientes se evitaron: $a$ ) informando a cada profesional de enfermería a que contestara exclusivamente sobre aquellos pacientes adscritos a su cupo y $b$ ) verificando que el código anonimizado creado para cada paciente no estuviese repetido. Los detalles de la aplicación de la encuesta según la iniciativa CHERRIES pueden verse en Material suplementario 2, iniciativa CHERRIES. Los datos de población adscrita a cada profesional fueron extraídos de tarjeta sanitaria del Servicio de Salud de Castilla-La Mancha (SESCAM), segregando por género, por centros de salud y por edad (menor de 14 años, 14-64 años y 65 o más años).

\section{Variables}

Se recogieron variables para estudiar la población incluida, el centro de salud de donde procedía la muestra, y variables para caracterizar las lesiones y el tratamiento empleado, ver Material suplementario 1, cuestionario Google Forms.

En UPP mayores de $10 \mathrm{~cm}^{2}$, el área de la úlcera se corrigió por el método de Kundin (área corregida $=$ largo $\times$ ancho $\times 0,785$ ) para evitar la sobreestimación ${ }^{12}$. En la categorización de la profundidad de las heridas ${ }^{1}$, y en consonancia con el GNEAUPP, las UPP de categoría 1 con más de 21 días de evolución o más de $20 \mathrm{~cm}^{2}$ de área se han recodificado a categoría 2 , al considerar que se trata de un error en la clasificación.

\section{Análisis de los datos}

La prevalencia puntual se calculó según la siguiente fórmula (expresada en tantos por mil):

- Prevalencia total (\%o): (n. ${ }^{\circ}$ de pacientes afectados a fecha 17 de febrero de 2020/total de pacientes adscritos a los profesionales que habían contestado la encuesta) $\times 1000$

Con ayuda de una calculadora online ${ }^{13}$ se calcularon intervalos de confianza del 95\% (IC95\%) para las prevalencias mediante el método de WiIson sin corrección por continuidad ${ }^{13}$, calculando además las prevalencias en función de la edad y el sexo. Las variables categóricas se presentaron mediante frecuencias y porcentajes, y las variables cuantitativas como media \pm desviación estándar (DE) o como mediana \pm amplitud intercuartil (AI) cuando aquellas no presentaban una distribución normal según la prueba de Kolmogorov-Smirnov. La asociación entre variables categóricas se realizó mediante la prueba de $\chi^{2}$ de Pearson, aplicando la corrección por continuidad de Yates si al menos el valor de una frecuencia esperada era menor que 5 y usando un nivel de significación de $p<0,050$. En el análisis estadístico se utilizaron los paquetes informáticos Epidat ${ }^{\oplus} 3.0$ e IBM ${ }^{\oplus}$-SPSS ${ }^{\oplus}-20$.

\section{Aspectos éticos}

El presente proyecto cuenta con el veredicto favorable de la Comisión de Investigación del Área de Salud de Cuenca (código 03032019) y del Comité de Ética de la GAI de Cuenca (código 2019/PI0319), cumpliendo con la Ley Orgánica 3/2018 de 5 de diciembre de Protección de datos de carácter personal y garantía de los derechos digitales y con la declaración de Helsinki. 


\section{$\checkmark$ RESULTADOS}

\section{Población de estudio}

La encuesta fue enviada al total de profesionales de enfermería de atención primaria de la GAI de Cuenca $(n=155$; población total adscrita=135107 habitantes). Asimismo, la encuesta fue contestada por 152 profesionales, consiguiendo una muestra final de estudio de 131190 habitantes (59,3\% procedencia urbana y $40,7 \%$ rural), lo que representa una tasa de respuesta del $98,1 \%$ y una precisión del $99,996 \%$ (nivel de confianza del $99 \%$, prevalencia de UPP previa estimada $0,109 \%^{2}$ ).

Un total de 51 pacientes presentaron UPP, de los que el 68,6\% eran mujeres y el 29,4\%, diabéticos. La mediana de edad fue de 89,0 años con $\mathrm{AI}=8,0$ ańos, y solo un 3,9\% de los pacientes presentó movilidad normal, mientras que el 90,2\% presentó incontinencia urinaria. Las características de los pacientes con UPP se muestran en la tabla 1.

\section{Prevalencias}

La prevalencia global de pacientes con UPP fue de 0,389\% (IC 95\%: $0,296-0,511$ ), siendo $0,532 \%$ en mujeres y $0,245 \%$ en hombres $(p=0,008)$ y aumentando la prevalencia con la edad en ambos géneros $(p<0,001$ para los tres rangos de edad). La tabla 2 muestra el número de pacientes afectados por UPP y las prevalencias (por mil) según edad y sexo.

\section{Tipología de las lesiones}

Se identificaron un total de 72 UPP, existiendo gran heterogeneidad en la superficie (mediana $=6,0, \mathrm{AI}=8,1 \mathrm{~cm}^{2}$ ) y tiempo de evolución (mediana $=60,0, A I=98,0$ días). Solo el 52,8\% de los pacientes presentó lesiones únicas, y una vez recodificadas, únicamente el 2,8\% de los pacientes mostró una categoría $=1$, mientras que el 16,7\% de los pacientes presentaba una categoría $=4$.

Asimismo, el 31,9\% eran lesiones recurrentes, existiendo evidencia de infección en el 9,8\% de las lesiones. La localización preferente de las lesiones fue el pie (36\%), el sacro/coxis (25\%) y el trocánter (18\%).
Tabla 1. Características de los pacientes con úlceras por presión

\begin{tabular}{|c|c|c|}
\hline & & $\begin{array}{c}\text { Total } \\
n=51\end{array}$ \\
\hline \multirow{2}{*}{ Edad } & Media (DE) & $86,6 \pm 10,4$ \\
\hline & Mediana (Al) & $89,0 \pm 8,0$ \\
\hline \multirow{2}{*}{ Género $(\%)$} & Hombre & $16(31,4)$ \\
\hline & Mujer & $35(68,6)$ \\
\hline \multirow{5}{*}{$\mathrm{DM}(\%)$} & Tipo 2 & $15(29,4)$ \\
\hline & Тіро 1 & - \\
\hline & Otras & - \\
\hline & No & $36(70,6)$ \\
\hline & No sabe & - \\
\hline \multirow{2}{*}{ HTA $(\%)$} & Sí & $27(52,9)$ \\
\hline & No & $24(47,1)$ \\
\hline \multirow{5}{*}{ Movilidad (\%) } & Normal & $2(3,9)$ \\
\hline & Encamado & $6(11,8)$ \\
\hline & Cama/sillón & $37(72,5)$ \\
\hline & Muletas/andador & $4(7,8)$ \\
\hline & Silla de ruedas & $2(3,9)$ \\
\hline \multirow{5}{*}{$\begin{array}{l}\text { Incontinencia } \\
\text { urinaria }(\%)\end{array}$} & No & $5(9,8)$ \\
\hline & Ocasional & $1(2,0)$ \\
\hline & Habitual & $6(11,8)$ \\
\hline & Pañal & $32(62,7)$ \\
\hline & Sonda vesical/colector & $7(13,7)$ \\
\hline \multirow{5}{*}{ Incontinencia fecal (\%) } & No & $16(31,4)$ \\
\hline & Ocasional & $5(9,8)$ \\
\hline & Habitual & $4(7,89$ \\
\hline & Pañal & $25(49,0)$ \\
\hline & No sabe & $1(2,0)$ \\
\hline
\end{tabular}

Al: amplitud intercuartil; DE: desviación estándar; DM: diabetes mellitus; HTA: hipertensión arterial; n: número de pacientes.

Los valores enteros se refieren a frecuencias y los valores entre paréntesis a porcentajes.

Tabla 2. Número de pacientes afectados por úlceras por presión y prevalencias (por mil) según género y edad

\begin{tabular}{|c|c|c|c|c|c|}
\hline & 0-14 años & 15-64 años & $\geq 65$ años & Total adulto ${ }^{\mathrm{a}}$ & Total \\
\hline Población & 15394 & 86543 & 29253 & 115796 & 131190 \\
\hline Hombre & 7806 & 44526 & 13109 & 57635 & 65401 \\
\hline Mujer & 7588 & 42017 & 16144 & 58161 & 65789 \\
\hline Casos (hombre) & 0 & 1 & 15 & 16 & 16 \\
\hline Casos (mujer) & 0 & 3 & 32 & 35 & 35 \\
\hline Total casos & 0 & 4 & 47 & 51 & 51 \\
\hline Prevalencia hombre $(\%)$ & 0 & 0,022 & 1,144 & 0,278 & 0,245 \\
\hline IC $95 \%$ & $0,000-0,492$ & $0,004-0,127$ & $0,694-1,887$ & $0,171-0,451$ & $0,151-0,397$ \\
\hline Prevalencia mujer (\%o) & 0 & 0,071 & 1,982 & 0,602 & 0,532 \\
\hline IC $95 \%$ & $0,000-0,506$ & $0,024-0,210$ & $1,404-2,797$ & $0,433-0,837$ & $0,383-0,740$ \\
\hline Prevalencia total $(\%)$ & 0 & 0,046 & 1,607 & 0,440 & 0,389 \\
\hline IC 95\% & $0,000-0,249$ & $0,018-0,119$ & $1,209-2,136$ & $0,335-0,579$ & $0,296-0,511$ \\
\hline$p$ & - & $0,577^{b}$ & 0,075 & 0,009 & 0,008 \\
\hline
\end{tabular}

a Población $\geq 15$ años.

bPrueba de $\chi^{2}$ con corrección de Yates; cuando no se indica nada, la prueba utilizada fue $\chi^{2}$ (el contraste de hipótesis indica la diferencia entre géneros para ese rango de edad).

IC: intervalo de confianza. 
Tabla 3. Características clínicas de las úlceras por presión

\begin{tabular}{|c|c|c|}
\hline & $\begin{array}{c}\text { UPP } \\
n=72\end{array}$ \\
\hline \multirow{2}{*}{$\begin{array}{l}\text { Tiempo de evolución } \\
\text { (días) }\end{array}$} & Media (DE) & $191,2 \pm 472,7$ \\
\hline & Mediana (Al) & $60,0 \pm 98,0$ \\
\hline \multirow{2}{*}{ Superficie $\left(\mathrm{cm}^{2}\right)$} & Media (DE) & $10,5 \pm 16,1$ \\
\hline & Mediana (Al) & $6,0 \pm 8,1$ \\
\hline \multirow{5}{*}{ Lesiones múltiples (\%) } & 1 & $38(52,8)$ \\
\hline & 2 & $18(25,0)$ \\
\hline & 3 & $12(16,7)$ \\
\hline & 4 & $4(5,6)$ \\
\hline & 5 & - \\
\hline \multirow{7}{*}{$\begin{array}{l}\text { Profundidad, } \\
\text { categoría (\%) }\end{array}$} & 1 no recodificada & $5(6,9)$ \\
\hline & 1 recodificada & $2(2,8)$ \\
\hline & 2 no recodificada & $26(36,1)$ \\
\hline & 2 recodificada & $29(40,3)$ \\
\hline & 3 & $19(26,4)$ \\
\hline & 4 & $12(16,7)$ \\
\hline & No sabe/no estadificable & $10(13,9)$ \\
\hline \multirow{3}{*}{ ¿Recurrente? (\%) } & Sí & $23(31,9)$ \\
\hline & No & $46(63,9)$ \\
\hline & No sabe & $3(4,2)$ \\
\hline \multirow{4}{*}{ ¿Infectada? (\%) } & Sí, signos clásicos & $4(5,6)$ \\
\hline & Sí, signos atípicos & $3(4,2)$ \\
\hline & No & $61(84,7)$ \\
\hline & No sabe & $4(5,6)$ \\
\hline
\end{tabular}

Al: amplitud intercuartil; DE: desviación estándar; n: número de lesiones; UPP: úlcera por presión. Profundidad (recodificación): las úlceras por presión de categoría 1 con más de 21 días de evolución o más de $20 \mathrm{~cm}^{2}$ de área se han recodificado a categoría 2, por considerar que se trata de un error en la clasificación. Los valores enteros se refieren a frecuencias y los valores entre paréntesis a porcentajes.

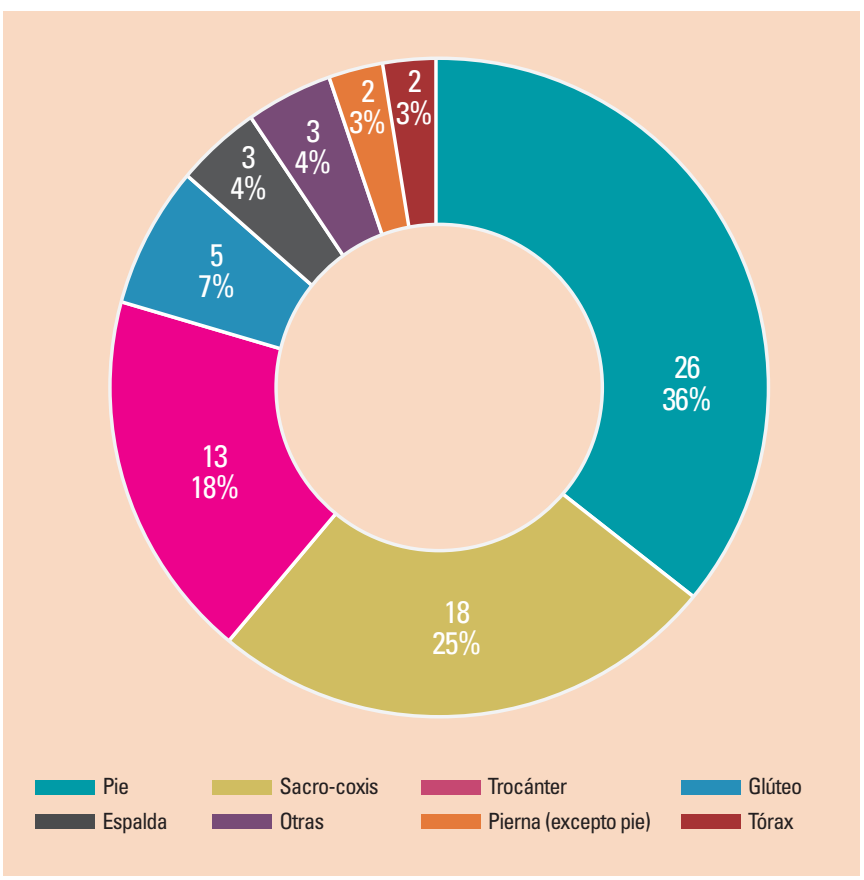

Figura 1. Localización de las distintas úlceras por presión.
La tabla 3 y la figura 1 muestran las características clínicas y la localización de las UPP, respectivamente.

\section{Tratamiento empleado}

En relación con la prevención, hasta el $31,4 \%$ de los profesionales no usó escalas de valoración del riesgo de UPP y hasta en el $25,5 \%$ de los pacientes no se usaba superficie especial para el manejo de la presión (SEMP). Sin embargo, en el 68,6\% de pacientes se aplicaban cambios posturales reglados. En el 60,8\% de los pacientes se usaron al menos dos diferentes estrategias de alivio de la presión, predominando los apósitos protectores de espuma/foam/hidrocoloide ( $47,1 \%$ de los pacientes), las taloneras de textil $(41,2 \%)$ y los ácidos grasos hiperoxigenados (AGHO) $(35,5 \%)$.

En relación con el tratamiento, se usó la cura en ambiente húmedo hasta en el 59,7\% de los casos, variando el material ampliamente con predilección por los apósitos hidrocelulares/espumas $(40,3 \%)$, hidrogeles $(23,6 \%)$ e hidrofibra de hidrocoloide con plata $(22,2 \%)$. Sin embargo, el uso de terapias avanzadas como la terapia de presión negativa (TPN) fue anecdótico, utilizándose solo en una lesión. La tabla 4 muestra las características del tratamiento y las medidas preventivas empleadas.

\section{У DISCUSIÓN}

Este trabajo representa el primer estudio de prevalencia de UPP específicamente desarrollado en centros de atención primaria de Cuenca, encontrando prevalencias bajas en comparación con otros estudios nacionales e internacionales.

A través de un cuestionario online a profesionales de enfermería, se analizó una muestra total de 131190 individuos, consiguiendo una tasa de respuesta del 98,1\%. Esto supone, junto con un estudio irlandés $(97,9 \%)^{14}$, una de las mayores tasas de respuesta encontradas en la literatura científica, muy lejos del 22,9\% conseguido por el GNEAUPP en su

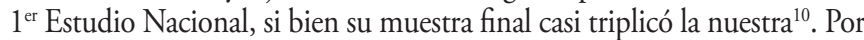
tanto, nuestro estudio analiza una muestra totalmente representativa que permite conocer con una alta precisión las UPP existentes en el contexto de atención primaria en una provincia con un alto componente rural como Cuenca.

La prevalencia global de UPP en nuestro estudio fue de 0,389\%o $(0,440 \%$ considerando población adulta), una cifra muy inferior a la encontrada por el GNEAUPP en su $4^{\circ}$ Estudio Nacional $(1,087 \%)^{2}$. No obstante, ambas cifras están dentro del rango encontrado en otras series internacionales que irían desde 0,168\%o en mujeres de América Central hasta 2,324\%o en mujeres del norte de África y del Medio Este ${ }^{15}$. Aunque puede ser difícil comparar nuestra atención primaria con la de esos países, el país vecino Portugal, en un estudio reciente, ha informado de una prevalencia del $0,2 \%$ en un contexto comunitario, si bien reconocen limitaciones en su estudio que podrían haber infraestimado la prevalen$\mathrm{cia}^{16}$. Lamentablemente, nuestro estudio no obtiene datos de prevalencia dentro de los programas de atención domiciliaria, información que sería muy útil al permitir una comparación directa menos sesgada con otros estudios, especialmente teniendo en cuenta que hasta el 50\% de las UPP están en sus domicilios ${ }^{17}$. La tendencia en la prevalencia de UPP no parece explicar nuestras bajas prevalencias, ya que los tres últimos estudios nacionales del GNEAUPP muestran prevalencias claramente crecientes $^{2,11,12}$. En este sentido, el 5 Estudio Nacional, del que se prevé una publicación inminente, podrá arrojar nuevos datos de tendencia.

Una posible explicación de esta baja prevalencia de UPP podría ser el gran componente rural, despoblación y dispersión geográfica que acompa- 
Tabla 4. Periodicidad, tipo de cura, apósitos y otras técnicas empleados en el tratamiento o prevención de las úlceras por presión

\begin{tabular}{|c|c|c|}
\hline & & $\begin{array}{l}\text { UPP } \\
n=72\end{array}$ \\
\hline \multirow{2}{*}{$\begin{array}{l}\text { Cura, periodicidad } \\
\text { (días) }\end{array}$} & Media (DE) & $2,64 \pm 1,3$ \\
\hline & Mediana (Al) & $2,50 \pm 1,0$ \\
\hline \multirow{3}{*}{ Cura, tipos (\%) } & Ambiente húmedo & $43(59,7)$ \\
\hline & Seca & $25(34,7)$ \\
\hline & Otros & $4(5,6)$ \\
\hline \multirow{9}{*}{ Apósito, tipos (\%) } & Hidrocelular/espuma & $29(40,3)$ \\
\hline & Gasa & $14(19,4)$ \\
\hline & $\begin{array}{l}\text { Hidrofibra hidrocoloide con } \\
\text { plata }\end{array}$ & $16(22,2)$ \\
\hline & Alginato & $13(18,1)$ \\
\hline & Silicona & $3(4,2)$ \\
\hline & Hidrogel & $17(23,6)$ \\
\hline & $\begin{array}{l}\text { Hidrofibra hidrocoloide sin } \\
\text { plata }\end{array}$ & $4(5,6)$ \\
\hline & Hidrocoloide & $5(6,9)$ \\
\hline & TPN & $1(1,4)$ \\
\hline \multirow{3}{*}{$\begin{array}{l}\text { Otros tratamientos } \\
(\%)\end{array}$} & Tul graso & $4(5,6)$ \\
\hline & Colagenasa & $2(2,8)$ \\
\hline & $\mathrm{AGHO}^{\mathrm{C}}$ & $2(2,8)$ \\
\hline \multirow{6}{*}{$\begin{array}{l}\text { Infección, } \\
\text { tratamiento } \\
(\%)\end{array}$} & Antibiótico sistémico & $1(14,3)$ \\
\hline & Antibiótico tópico & $1(14,3)$ \\
\hline & Apósito con plata & $4(57,1)$ \\
\hline & Otros & $2(18,6)$ \\
\hline & $\begin{array}{l}\text { Combinación de varios } \\
\text { métodos }\end{array}$ & $2(18,6)$ \\
\hline & Sin tratamiento & $1(14,3)$ \\
\hline \multirow{5}{*}{$\begin{array}{l}\text { Uso de SEMPb } \\
(\%)\end{array}$} & Estática & $3(5,9)$ \\
\hline & Dinámica & $17(33,3)$ \\
\hline & Ambas & $7(13,7)$ \\
\hline & No & $13(25,5)$ \\
\hline & No sabe & $11(21,6)$ \\
\hline \multirow{5}{*}{$\begin{array}{l}\text { Escalas de } \\
\text { valoración }{ }^{b} \\
(\%)\end{array}$} & Braden & $10(19,6)$ \\
\hline & Norton & $8(15,7)$ \\
\hline & Norton modificada & $2(3,9)$ \\
\hline & No usa & $16(31,4)$ \\
\hline & No sabe & $15(29,4)$ \\
\hline \multirow{5}{*}{$\begin{array}{l}\text { Manejo de la } \\
\text { presiónd }^{\text {d }} \\
(\%)\end{array}$} & Cambios posturales & $35(68,6)$ \\
\hline & AGHO & $18(35,3)$ \\
\hline & Espuma/foam/hidrocoloide & $24(47,1)$ \\
\hline & Taloneras de textil & $21(41,2)$ \\
\hline & No sabe & $8(15,7)$ \\
\hline
\end{tabular}

a Los porcentajes se calculan sobre las lesiones que presentaban infección.

'Los datos se refieren al porcentaje de pacientes (no al de lesiones).

${ }^{c}$ AGHO utilizado como tratamiento, no como preventivo.

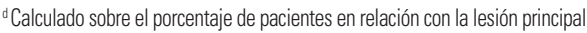

Los valores enteros se refieren a frecuencias y los valores entre paréntesis, a porcentajes.

AGHO: ácido graso hiperoxigenado; Al: amplitud intercuartil; DE: desviación estándar; n: número de lesiones; SEMP: superficie especial para el manejo de la presión; TPN: terapia de presión negativa; UPP: úlcera por presión. ñan a una provincia como Cuenca, claro exponente de la denominada "España vacía”. Así, en nuestra GAI, aunque el $84,4 \%$ de los centros de salud eran rurales, estos solo atendían al 40,7\% de la población total. Las mencionadas bajas prevalencias concordarían con el hallazgo del GNEAUPP de su $4^{\circ}$ Estudio Nacional que encontró una prevalencia estadísticamente significativa menor en centros rurales frente a urbanos en las UPP $(6,79 \%$ y $9,50 \%$, respectivamente $)^{2}$. Además, a nivel nacional, un estudio realizado en otra región con alto componente rural, La Rioja, encontró prevalencias en atención primaria de 2,600\%, que no son muy distintas a las halladas en nuestro estudio en ese rango de edad $(1,607 \% 0)^{17}$. Asimismo, el alto ratio enfermera/paciente encontrado en Cuenca con respecto al global nacional (1,1 por mil pacientes frente a 0,6 por mil pacientes en España, según el Ministerio de Sanidad), o el mayor desarrollo de los cuidados familiares y las estructuras de autocuidados informales de las zonas rurales ${ }^{18}$ podrían explicar las bajas prevalencias encontradas.

En paralelo, la completa titularidad pública de los centros de salud estudiados también podría haber influido en nuestra baja prevalencia. Así, el $4^{\circ}$ Estudio Nacional del GNEAUPP encontró en los centros sociosanitarios públicos una prevalencia de UPP menor que en los privados o concertados, si bien existían pocos datos de centros privados, y no había datos directos de atención primaria ${ }^{2}$. Por otro lado, aunque nuestro estudio se circunscribe a la GAI de Cuenca, fue realizado enteramente por profesionales asistenciales enfermeros, sin existir ningún sesgo institucional que pudiera infraestimar las prevalencias encontradas.

A pesar de las bajas prevalencias, el perfil de gravedad de las lesiones de nuestro estudio fue similar al encontrado en el $4^{\circ}$ Estudio Nacional del GNEAUPP $^{2}$, hallando idéntica superficie de las UPP, y parecidas tasas de recurrencia y categorización de las heridas, aunque con un tiempo de evolución mayor que en el estudio del GNEAUPP (60 vs. 45 días, respectivamente) $)^{2}$.

Aunque existe evidencia de que las UPP no son exclusivas de la tercera edad $^{19}$, nuestro estudio encontró que la prevalencia se incrementaba de forma significativa al aumentar la edad de los pacientes. Al igual que notificó el

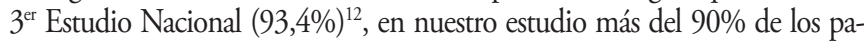
cientes con UPP tenían una edad igual o superior a 65 años y tal como ocurría en el $4^{\circ}$ Estudio Nacional, la prevalencia en mayores de 65 años cuadruplicó la de la población general ${ }^{2}$. Ello pone de relieve el perfil de alta fragilidad que generalmente se asocia a las UPP ${ }^{16,20}$, especialmente en el contexto de atención primaria. Así, solo el 9,8\% de los pacientes con UPP era continente a nivel vesical y más de un 70,0\% estaba postrado en cama/sillón.

En lo relativo al género, en nuestro estudio las UPP afectaron de forma predominante a las mujeres (68,6\% vs. $31,4 \%)$. Este hallazgo concuerda con los tres estudios nacionales que de forma sistemática encontraron mayores prevalencias de UPP en mujeres en atención primaria (64,2\%, 63,7\% y $62,7 \%$ para el $2^{\circ}, 3^{\text {er }}$ y $4^{\circ}$ Estudio Nacional, respectivamente $\left.{ }^{2,11,12}\right)$, quizá debido a una peor movilidad de las mujeres en relación con mayores tasas de obesidad ${ }^{21}$ o a una mayor esperanza de vida. Sin embargo, la presencia del género femenino como factor de riesgo de UPP no ha sido confirmada en otros estudios. Así, un estudio encontró que el género masculino era un factor predictor de ulceración en pacientes comunitarios que recibían cuidados familiares ${ }^{22}$, y otro estudio encontró prevalencias similares en ambos sexos en el contexto de UPP adquiridas en el hospital o residencias sociosanitarias, si bien el mayor uso de las SEMP en las mujeres pudo influir en los resultados ${ }^{23}$.

A nivel de prevención, al igual que notificó el GNEAUPP en el $3^{\text {er }}$ Estudio Nacional, la escala Braden (19,6\%) fue la más utilizada en nuestro estudio, seguida muy de cerca por la escala de Norton (15,7\%), pero llama la atención que hasta el $31,4 \%$ de los profesionales no usaba ninguna escala. Análogamente, aunque el uso de las SEMP se ha incrementado notablemente en relación con el $1^{\text {er }}$ Estudio Nacional, existe una infrautilización si nos comparamos con el $4^{\circ}$ Estudio del GNEAUPP (52,9\% vs. 65,0\%). 
Además, se usaron pocos AGHO en la profilaxis de la UPP y el uso de la TPN fue anecdótico, lo que podría estar en relación con la falta de financiación de estos materiales y la inexistencia de un banco de material para préstamos como existe en otras comunidades. No obstante, pensamos que en los estudios nacionales pudiera haber cierto sesgo de respuesta, ya que la mayoría de los cuestionarios son enviados a miembros del GNEAUPP (profesionales con un alto conocimiento y motivación en el cuidado de heridas), potencialmente sobreestimando el uso de terapias específicas avanzadas, algunas poco conocidas en ámbitos no expertos. Quizá nuestro estudio refleje de forma más adecuada la situación real en atención primaria, al implicar a la práctica totalidad de la enfermería de nuestros centros de salud.

\section{$\searrow$ LIMITACIONES}

Aunque nuestro estudio cumple con la mayoría de los criterios de calidad establecidos para este tipo de estudios ${ }^{24-26}$, dos aspectos podrían ser fuente de $\left.\operatorname{sesgo}^{27}: 1\right)$ el grado de conocimiento y por tanto la precisión en el diagnóstico de las UPP por enfermería pudo no ser homogéneo y 2) el conocimiento por parte del equipo investigador de los profesionales que contestaban la encuesta (vía correo corporativo) podría haber incurrido en cierto sesgo de complacencia por parte de aquellos. Además, en nuestro estudio no se discriminó entre lesiones cutáneas asociadas a la humedad (LESCAH) y UPP, por lo que se asume que la prevalencia de UPP podría estar algo sobreestimada al incluir las LESCAH.

\section{У CONCLUSIÓN}

Este estudio encontró que la prevalencia de UPP en una provincia con alto componente rural fue baja, si bien la distribución por género y edad y el perfil de las lesiones guarda analogía con el de otros estudios nacionales. Asimismo, el uso de estrategias preventivas y de tratamientos tecnológicamente avanzados continúa siendo bajo.

Se hacen necesarios más estudios para conocer hasta qué punto las prevalencias de UPP encontradas previamente pueden haber sido sobreestimadas en la Espańa rural

\section{Conflicto de intereses}

Los autores declaran no tener conflicto de intereses en relación con el presente proyecto.

\section{Financiación}

El presente estudio no cuenta con ningún tipo de financiación.

\section{Agradecimientos}

Los autores agradecen la colaboración de los pacientes adscritos a los centros de salud de la Gerencia de Atención Integrada de Cuenca, y la implicación de los profesionales de enfermería de dicha Gerencia. Asimismo, los autores agradecen la colaboración de todo el Grupo de Trabajo en Heridas de la Gerencia de Atención Integrada de Cuenca por sus interesantes comentarios.

\section{VIBLIOGRAFÍA}

1. National Pressure Ulcer Advisory Panel, European Pressure Ulcer Advisory Panel, Pan Pacific Pressure Injury Alliance. Prevención y tratamiento de las úlceras por presión: Guía de consulta rápida. En: Haesler E, ed. Petrh, Australia; Cambirdge media; 2014. Versión española.

2. Pancorbo-Hidalgo PL, García-Fernández FP, Torra i Bou J-E, Verdú Soriano J. Soldevilla-Agreda JJ. Epidemiología de las úlceras por presión en España en 2013: $4^{\circ}$ Estudio Nacional de Prevalencia. Gerokomos. 2014;25(4):162-70.

3. Bauer K, Rock K, Nazzal M, Jones 0, Ou W. Pressure Ulcers in the United States' Inpatient Population From 2008 to 2012: Results of a Retrospective Nationwide Study. Ostomy Wound Manage. 2016;62(11):30-8

4. Verdú J, Nolasco A, García C. Análisis y evolución de la mortalidad por úlceras por presión en España. Período 1987-1999. Gerokomos. 2003;14(4):212-26

5. Essex HN, Clark M, Sims J, Warriner A, Cullum N. Health-related quality of life in hospital inpatients with pressure ulceration: assessment using generic health-related quality of life measures. Wound Repair Regen. 2009:17(6):797-805

6. García-Sánchez FJ, Martínez-Vizcaíno V Rodríguez-Martín B. Barriers and facilitators for caregiver involvement in the home care of people with pressure injuries: A qualitative study. PLoS One. 2019;14(12):e0226359

7. Posnett J, Gottrup F, Lundgren H, Saal G. The resource impact of wounds on health-care providers in Europe. J Wound Care. 2009:18(4):154-61.

8. Soldevilla Agreda JJ, Torra I Bou JE, Posnett J, Verdu Soriano J, San Miguel L, Mayan Santos M. The Burden of Pressure Ulcers in Spain. Wounds. 2007;19(7):201-6.

9. Martinengo L, Olsson M, Baipai R, Soljak M, Upton Z, Schmidtchen $A$, et al. Prevalence of chronic wounds in the general population: systematic review and meta-analysis of observational studies. Ann Epidemiol. 2019;29:8-15.
10. Torra i Bou JE, Rueda López J, Soldevilla Agreda JJ, Martínez Cuervo F, Verdú Soriano J. Primer Estudio Nacional de Prevalencia de Úlceras por Presión en España. Epidemiología y variables definitorias de las lesiones y pacientes. Gerokomos. 2003;14 (1):37-47.

11. Soldevilla Agreda JJ, Torra i Bou J-E, Verdú Soriano J, Martínez Cuervo F López Casanova P Rueda López J et al . $2^{0}$ Estudio $\mathrm{Na}$ cional de Prevalencia de Úlceras por Presión en España, 2005: Epidemiología y variables definitorias de las lesiones y pacientes. Gerokomos. 2006:17(3):154-72.

12. Soldevilla Agreda JJ, Torra i Bou J-E, Verdú Soriano J, López

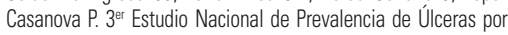
Presión en España, 2009: Epidemiología y variables definitorias de las lesiones y pacientes. Gerokomos. 2011:22(2):77-90.

13. Herbert R. Confidence Interval Calculator (2013). https://www. pedro.org.au/spanish/downloads/confidence-interval-calculator/. (Acceso 01 de septiembre de 2020)

14. O'Brien JF, Grace PA, Perry IJ, Burke PE. Prevalence and etiology of I eg ulcers in Ireland. Ir J Med Sci. 2000;169:110-2.

15. Hay RJ, Johns NE, Williams HC, Bolliger IW, Dellavalle RP, Margolis DJ, et al. The global burden of skin disease in 2010: an analysis of the prevalence and impact of skin conditions. J Invest Dermatol. 2014;134(6):1527-34.

16. Lopes TS, Videira LMMD, Saraiva DMRE, Agostinho ES, Bandarra AJF. Multicentre study of pressure ulcer point prevalence in a Portuguese region. J Tissue Viability. 2020;29(1):12-8.

17. Soldevilla Agreda JJ, Torra i Bou JE. Epidemiología de las úlceras por presión en España. Estudio piloto en la comunidad autónoma de la Rioja. Gerokomos. 1999;10(2):75-86.

18. Elizalde-San Miguel B. El cuidado informal en las zonas rurales. Nuevas formas, nuevos actores. Social and Education History. 2017:6(2):168-95.

19. Delmore B, VanGilder C, Koloms K, Ayello EA. Pressure Injuries in the Pediatric Population: Analysis of the 2008-2018 International
Pressure Ulcer Prevalence Survey Data. Adv Skin Wound Care. 2020;33(6):301-6.

20. Pokorná A, Benešová K, Jarkovský J, Mužík J, Beeckman D. Pressure Injuries in Inpatient Care Facilities in the Czech Republic: Analysis of a National Electronic Database. J Wound Ostomy Continence Nurs. 2017:44(4):331-5.

21. Hyun S, Li X, Vermillion B, Newton C, Fall M, Kaewprag P, et al. Body mass index and pressure ulcers: improved predictability of pressure ulcers in intensive care patients. Am J Crit Care. 2014;23(6):494-500.

22. Bergquist S, Frantz R. Pressure ulcers in community-based olde adults receiving home health care. Prevalence, incidence, and as sociated risk factors. Adv Wound Care. 1999:12(7):339-51.

23. Lichterfeld-Kottner A, Lahmann N, Kottner J. Sex-specific differences in prevention and treatment of institutional-acquired pressure ulcers in hospitals and nursing homes. J Tissue Viability. 2020;29(3):204-10.

24. Shamliyan TA, Kane RL, Ansari MT, et al. Development of Quality Criteria To Evaluate Nontherapeutic Studies of Incidence, Prevalence, or Risk Factors of Chronic Diseases: Pilot Study of New Checklists [Internet]. Rockville (MD): Agency for Healthcare Research and Quality (US); 2011 Jan. Disponible en: https://www. ncbi.nlm.nih.gov/books/NBK53272/

25. Vandenbroucke JP, von Elm E, Altman DG, Gøtzsche PC, Mulrow CD, Pocock SJ, et al. Strengthening the Reporting of Observational Studies in Epidemiology (STROBE): explanation and elaboration. Int J Surg. 2014;12(12):1500-24.

26. Munn Z, Moola S, Riitano D, Lisy K. The development of a critica appraisal tool for use in systematic reviews addressing questions of prevalence. Int J Health Policy Manag. 2014;3(3):123-8.

27. Loney PL, Chambers IW, Bennett KJ, Roberts JG, Stratford PW. Critical appraisal of the health research literature: prevalence or incidence of a health problem. Chronic Dis Can 1998;19(4):170-6 